\title{
Efficient Support for Matrix Computations on Heterogeneous Multi-core and Multi-GPU Architectures
}

\author{
Fengguang Song \\ University of Tennessee \\ EECS Department \\ Knoxville, TN, USA \\ song@eecs.utk.edu
}

\author{
Stanimire Tomov \\ University of Tennessee \\ EECS Department \\ Knoxville, TN, USA \\ tomov@eecs.utk.edu
}

\author{
Jack Dongarra \\ University of Tennessee \\ Oak Ridge National Laboratory \\ University of Manchester \\ dongarra@eecs.utk.edu
}

\begin{abstract}
We present a new methodology for utilizing all CPU cores and all GPUs on a heterogeneous multicore and multi-GPU system to support matrix computations efficiently. Our approach is able to achieve the objectives of a high degree of parallelism, minimized synchronization, minimized communication, and load balancing. Our main idea is to treat the heterogeneous system as a distributed-memory machine, and to use a heterogeneous 1-D block cyclic distribution to allocate data to the host system and GPUs to minimize communication. We have designed heterogeneous algorithms with two different tile sizes (one for CPU cores and the other for GPUs) to cope with processor heterogeneity. We propose an auto-tuning method to determine the best tile sizes to attain both high performance and load balancing. We have also implemented a new runtime system and applied it to the Cholesky and QR factorizations. Our experiments on a compute node with two Intel Westmere hexa-core CPUs and three Nvidia Fermi GPUs demonstrate good weak scalability, strong scalability, load balance, and efficiency of our approach.
\end{abstract}

\section{INTRODUCTION}

As the performance of both multicore CPU and GPU continues to scale at a Moore's law rate, it is becoming pervasive to use heterogeneous multicore and multi-GPU architectures to attain the highest performance possible from a single compute node. Before making parallel programs run efficiently on a distributed-memory system, it is critical to achieve high performance on a single node first. However, the heterogeneity in the multi-core and multi-GPU architecture has introduced new challenges to algorithm design and system software.

Over the last few years, our colleagues at the University of Tennessee have developed the PLASMA library 2 to solve linear algebra problems on multicore architectures. In parallel with PLASMA, we have also developed another library called MAGMA 27] to solve linear algebra problems on GPUs. While PLASMA and MAGMA aim to provide the same routines as LAPACK [4], one is used for multicore CPUs, and the other for a single core with an attached GPU, respectively. Our goal is to utilize all cores and all GPUs efficiently on a single multicore and multi-GPU system to support matrix computations.

\footnotetext{
* This material is based upon work supported by the NSF grants CCF-0811642, OCI-0910735, by the DOE grant DEFC02-06ER25761, and by Microsoft Research.
}

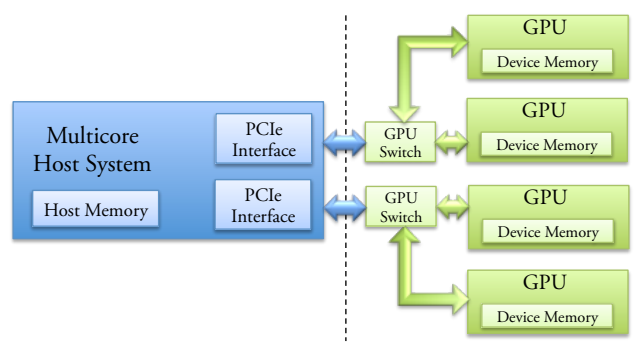

Figure 1: An example of a heterogeneous multi-core and multi-GPU system. The host system is connected to four GPUs via two PCI Express connections. The host system and the GPUs have separate memory spaces.

Figure 1 shows the architecture of a heterogeneous multicore and multi-GPU system we are considering. The multicore host system is connected to four GPUs via two PCI Express connections and each pair of GPUs share a GPU switch. To design new software on this type of heterogeneous architectures, we must consider the following special features: (1) The host and the GPUs have different memory spaces and an explicit memory copy is required to transfer data between the host and a GPU; (2) The system is also different from a distributed-memory machine since each GPU is actually controlled by a thread running on the host (more like pthreads on a shared-memory machine); (3) The processor heterogeneity between CPUs and GPUs; (4) GPUs are optimized for throughput and expect a larger input size than CPUs which are optimized for latency [24]; (5) As the performance gap between a GPU and its PCI-Express interconnection to the host becomes larger, network is eventually the bottleneck for the entire system. In this work, we take into account all these factors and strive to meet the following objectives in order to obtain high performance: a high degree of parallelism, minimized synchronization, minimized communication, and load balancing. We propose to design new heterogeneous algorithms and to use a simple but practical static data distribution to achieve the objectives simultaneously.

This paper describes heterogeneous rectangular tile algorithms with hybrid tile sizes, heterogeneous 1-D block cyclic data distribution, a new runtime system, and an auto-tuning method to determine the hybrid tile sizes. The rectangular tile algorithms build upon the previous tile algorithms, which divide a matrix into square tiles and exhibit a high degree of parallelism and minimized synchronizations 13,14 
(Section 2.1 introduces the tile algorithms briefly). However, a unique tile size does not work well for both CPU cores and GPUs at the same time (either too small or too big). A big tile will clobber a CPU core and a small tile cannot attain high performance on a GPU. Therefore, we have redesigned the tile algorithms so that they consist of two types of tiles: smaller tiles suitable for CPU cores and bigger tiles suitable for GPUs. For instance, Fig. 2 depicts two matrices consisting of a set of small and big rectangular tiles. The rectangular tile algorithms execute in a fashion similar to the tile algorithms such that whenever a task computing a tile at $[I, J]$ is completed, it will trigger new tasks on the right hand side of the $J$-th tile column and below the $I$-th tile row. Here the rectangle tile at $[I, J]$ can be either small or big, and is different from the tile algorithms.

We regard the multicore and multi-GPU system as a distributed memory machine and place greater emphasis on communication minimization. We statically store small rectangular tiles on the host and big rectangular tiles on the GPUs respectively to cope with processor heterogeneity and reduce data movement. In order to distribute the small and big rectangular tiles to the host and GPUs evenly, we propose a heterogeneous 1-D column block cyclic distribution method. The basic idea is that we first map a matrix to only GPUs using a 1-D column block cyclic distribution, then we cut a slice from each block and assign it to the host system. Our analysis shows that the static distribution method is able to reach a near lower bound communication volume. We also propose an auto-tuning method to determine the best slice size to be cut from each block for load balancing.

We have designed a runtime system to support dynamic scheduling on the heterogeneous CPU+GPU system. The runtime system allows our programs to be executed in a data-availability-driven model where a parent task always tries to trigger its children. In order to address the specialties of the heterogeneous system, we have implemented a number of techniques to extend a centralized runtime system to a new one that also considers the machine a distributed memory system. The new runtime system is "hybrid" in the sense that its scheduling and computing components are centralized and resident in the single host system, but its data, pools of buffers, communication components, and task queues are distributed in the host and different GPUs.

We conducted experiments on the Keeneland system at the Oak Ridge National Laboratory. On a compute node with two Intel Westmere hexa-core CPUs and three Nvidia Fermi GPUs, both our Cholesky factorization and QR factorization exhibit scalable performance. In terms of weak scalability, we can attain a nearly constant Gflops/core and Gflops/GPU performance from 1 core to 9 cores+3 GPUs. And in strong scalability, we can reduce the execution time by two orders of magnitude from 1 core to 9 cores +3 GPUs.

To our best knowledge, this is the first work to consider the multicore and multi-GPU system a distributed-memory machine to minimize communication. Our work makes the following contributions: (i) new heterogeneous rectangular tile algorithms with hybrid tiles to handle processor heterogeneity, (ii) a heterogeneous 1-D block cyclic distribution based on a novel two-level partitioning scheme, (iii) an auto-tuning method to achieve load balancing, (iv) and a new runtime system to accommodate the special features of the heterogeneous system (i.e., a hybrid of a shared- and distributed-memory system).

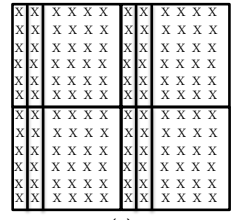

(a)

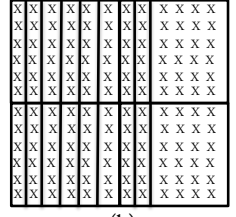

(b)
Figure 2: Matrices consisting of a mix of small and big rectangular tiles. (a) A $12 \times 12$ matrix is divided into 8 small tiles and 4 big tiles. (b) A $12 \times 12$ matrix is divided into 16 small tiles and 2 big tiles.

The rest of this paper is structured as follows. Section 2 explains the motivations for our work in more detail. Section 3 presents the heterogeneous rectangular tile algorithms. Section 4 describes the implementation and the auto-tuning method. Section 5 shows the experimental results. Section 6 presents related work. Section 7 summarizes our work.

\section{MOTIVATIONS AND BACKGROUND}

This section first introduces what the tile algorithms 14 are, then describes the motivations for our optimizations on GPUs and the reasons for choosing a static distribution method over a dynamic load balancing method.

\subsection{Tile Algorithms}

A tile algorithm divides an $n \times n$ matrix $A$ into a number of small $b \times b$ submatrices (aka "tiles") such that $A$ consists of $n_{b} \times n_{b}$ tiles, where $n_{b}=\frac{n}{b}$. $A$ can be expressed as follows:

$$
\left(\begin{array}{cccc}
A_{1,1} & A_{1,2} & \ldots & A_{1, n_{b}} \\
A_{2,1} & A_{2,2} & \ldots & A_{2, n_{b}} \\
\vdots & \vdots & \ddots & \vdots \\
A_{n_{b}, 1} & A_{n_{b}, 2} & \ldots & A_{n_{b}, n_{b}}
\end{array}\right)
$$

where $A_{i, j}$ is a $b \times b$ tile. In the tile algorithm, every task works on a small tile so that at any time there are a great amount of tasks available to execute. This way we can increase a program's thread level parallelism, which is desirable on multicore architectures. Take the tile QR factorization as an example. At the first iteration, the algorithm computes a QR factorization for $A_{1,1}$. The output of $A_{1,1}$ is then used to update the set of tiles on $A_{1,1}$ 's right hand side in an embarrassingly parallel way (i.e., $A_{1,2}, A_{1,3}, \ldots, A_{1, n_{b}}$ ). As soon as a tile-update in the $i$-th row completes, its neighbor below in the $(i+1)$-th row can start immediately. One could visualize the execution as falling columns of dominos from top to bottom. After updating all tiles in the $n_{b}$-th row, tile QR will continue to apply the same steps to the trailing submatrix $A_{2: n_{b}, 2: n_{b}}$ recursively 14. Our work extends the tile algorithms to rectangular tile algorithms to accommodate the processor heterogeneity between CPUs and GPUs.

\subsection{Optimization Issues on GPUs}

Although computational performance can be increased by adding more cores to a GPU, it is much more difficult to increase the network performance at the same rate. For instance, it has taken three years to introduce the PCI Express 3.0 Base specification to double its predecessor's bandwidth. We also expect the ratio of computational performance over communication bandwidth on a GPU will continue to increase. Hence, one of our objectives is to minimize commu- 


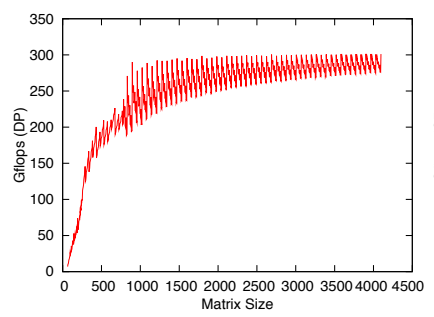

(a) Double precision

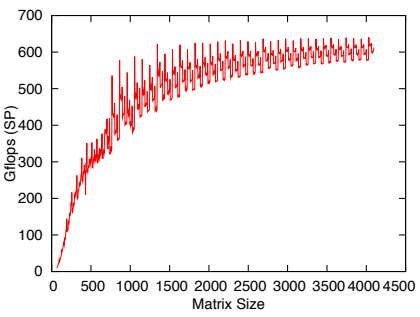

(b) Single precision
Figure 3: Matrix multiplication with CUBLAS 3.2 on an Nvidia Fermi M2070 GPU. (a) The maximum performance in double precision is 302 Gflops and the distance between the peaks is 64 . (b) The maximum performance in single precision is 622 Gflops and the distance between the peaks is 96 .

nication. A number of existing algorithms on distributedmemory supercomputers have addressed the issue to minimize communication. In ScaLAPACK 10], the parallel Cholesky, QR, and LU factorizations have been proven to reach the communication lower bound to within a logarithmic factor $8,16,18$. This has inspired us to adapt these efficient methods to optimize communication on the new multicore and multi-GPU systems.

Another issue is that a GPU cannot reach its high performance until given a sufficiently large input size. Figure 3 shows the performance of matrix multiplication on an Nvidia Fermi GPU using CUBLAS 3.2 in double precision and single precision, respectively. In general, the bigger the matrix size, the better the performance is, but the double precision matrix multiplication does not reach $95 \%$ of its maximum performance $(\max =302$ Gflops $)$ until the matrix size $N \geq 1088$. In single precision, it does not reach $95 \%$ of its maximum ( $\max =622$ Gflops) until $N \geq 1344$. Unlike a GPU, it is common for a CPU core to reach $90 \%$ of its maximum when $N \geq 200$ for matrix multiplications. However, solving a big matrix of size $N>1000$ by a single core is much slower than dividing it into smaller blocks and solving them in parallel by multiple cores. One could still use several cores to solve the big matrix in a fork-join manner, but it will introduce additional synchronization overhead and more CPU idle time 3, 12, 14. Therefore, we are motivated to design new heterogeneous algorithms to expose different tile sizes suitable for CPUs and GPUs, respectively.

\subsection{Using a Static Distribution Strategy}

We have employed a static strategy to distribute data and tasks to the host and different GPUs because of its less scheduling overhead, provably good communication cost, and simpler implementation than a dynamic strategy. The dynamic strategy often results in a sophisticated dynamic load balancing problem where a runtime system is required to monitor the status of CPUs and GPUs, and to measure the performance of different tasks on a CPU or a GPU to maintain load balancing. In consideration of additional communication optimization, scheduling policies, and related software cache mechanism, the dynamic load balancing problem becomes more challenging. Also our evaluation on QR factorizations (not reported here) shows that the static method is faster than the dynamic one for relatively small matrices (e.g., 60\% faster given a matrix of size 5760 and using four GPUs). In addition, to solve an $n \times n$ matrix, the software cache size on a GPU must be close to $\frac{n^{2}}{P}$ in order to reach an optimal communication volume proved by Theorem 1 .

THEOREM 1 ([20]). The communication volume of the classic matrix multiplication algorithm is equal to $\Omega\left(\frac{n^{3}}{P \sqrt{M}}\right)$, where $n$ is the matrix size, and $M$ is the local memory size on each of $P$ processes. When $M=O\left(\frac{n^{2}}{P}\right)$, the communication volume $\Omega\left(\frac{n^{2}}{\sqrt{P}}\right)$ is optimal.

Note that the communication volume of matrix multiplication is also the lower bound for other $\Theta\left(n^{3}\right)$ matrix computations such as Cholesky, QR, and LU factorizations. By setting the software cache size on each GPU as large as $O\left(\frac{n^{2}}{P}\right)$, the caching scheme will be the same as a static $1 \mathrm{D}$ or $2 \mathrm{D}$ block distribution method regarding memory usage. Also the caching scheme requires a good cache replacement mechanism. Later Section 3.5 shows that using a static distribution method can guarantee a near lower bound communication volume. To optimize both computation and communication without resorting to complex scheduling policies and software caches, we chose to use the simple static distribution strategy for the domain of matrix computations.

\section{HETEROGENEOUS RECTANGULAR TILE ALGORITHMS}

We extend the tile algorithms [14 to heterogeneous rectangular tile algorithms and apply them to the Cholesky and QR factorizations. We also introduce a novel two-level partitioning method and a heterogeneous 1-D column block cyclic distribution to map tiles and tasks to the host and GPUs to minimize communication.

\subsection{Hybrid-Size Rectangular Tiles}

The heterogeneous rectangular tile algorithm divides a matrix into a mix of small and big rectangular tiles. Figure 2 depicts two matrix examples that are divided into hybrid rectangular tiles. The two matrices have the same dimension but consist of a different number of small and big tiles.

However, the way to divide a matrix into rectangular tiles is not arbitrary. Constrained by the correctness of the algorithm, rectangular tiles must be aligned with each other and located in a collection of rows and columns. Their dimensions, however, could vary row by row or column by column (e.g., a row of tall tiles followed by a row of short tiles). Since we target a heterogenous system with two types of processors (i.e., CPU and GPU), we use two tile sizes: a small one for CPU and a big one for GPU. It should be easy to extend the algorithm to include more tile sizes.

On a heterogeneous multicore and multi-GPU system, we propose to use the following two-level partitioning scheme to create small tiles and big tiles: (1) At the top level, we divide a matrix into large square tiles of size $B \times B$; (2) Then we subdivide each top-level tile of size $B \times B$ into a number of small rectangular tiles of size $B \times b$ and a remaining tile. We use this scheme because it not only results in a clean code structure but also allows us to use a simple auto-tuning method to achieve load balancing. For instance, as shown in Fig. 2 (a), we first divide the $12 \times 12$ matrix into four $6 \times 6$ tiles, then we divide each $6 \times 6$ tile into two $6 \times 1$ and one $6 \times 4$ rectangular tiles. How to partition the top-level large tiles is dependent on the performance of the host and the performance of each GPU. Section 4.2 will introduce a method to determine an appropriate partitioning. 

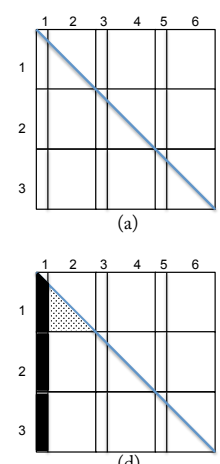
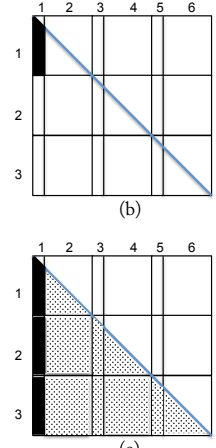
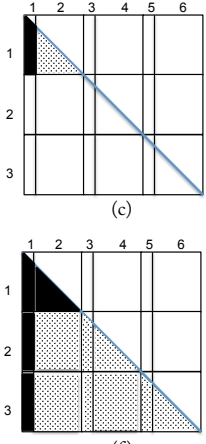
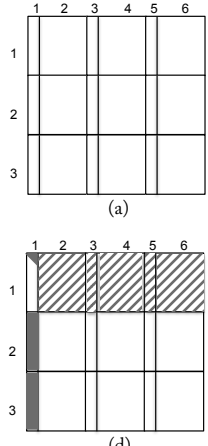

(d)
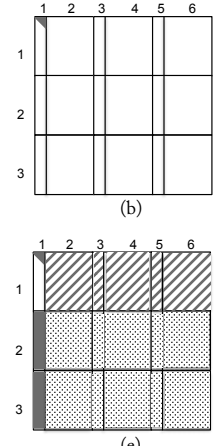
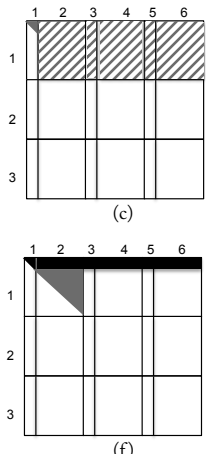

Figure 4: The operations of heterogeneous rectangular tile Cholesky factorization. (a) The symmetric positive definite matrix A. (b) Compute POTF2' to solve $L_{11}$. (c) Apply $L_{11}$ to update its right $A_{12}$ by matrix multiplication. (d) Compute TRSMs for all tiles below $L_{11}$ to solve $L_{21}$ and $L_{31}$. (e) Apply GSMMs to update all tiles on the right of TRSMs. (f) At the 2nd iteration, we repeat performing (b), (c), (d), (e) on the trailing submatrix that starts from the 2 nd tile column.

\subsection{Rectangular Tile Cholesky Factorization}

Given a matrix A of size $n \times n$ and two tile sizes of $\mathrm{B}$ and b, A can be expressed as follows:

$$
\left(\begin{array}{cc|cc|c}
\overbrace{a_{11}} & a_{12} \ldots A_{1 s} & \overbrace{a_{1(s+1)}} & a_{1(s+2)} \ldots A_{1(2 s)} & \ldots \\
a_{21} & a_{22} \ldots A_{2 s} & a_{2(s+1)} & a_{2(s+2)} \ldots A_{2(2 s)} & \ldots \\
\vdots & & \vdots & & \\
a_{p 1} & a_{p 2} \ldots A_{p s} & a_{p(s+1)} & a_{p(s+2)} \ldots A_{p(2 s)} & \ldots
\end{array}\right) \text {, where }
$$

an $\overbrace{a_{i(k s+1)} a_{i(k s+2)} \ldots A_{i(k s+s)}}$ forms a large tile of size $B \times B$. Here $a_{i j}$ represents a small rectangular tile of size $B \times b$, and $A_{i j}$ represents a tile of size $B \times(B-b(s-1))$ which is usually larger. We also assume $n=p B$ and $B>b$.

Algorithm 1 shows the heterogeneous rectangular tile Cholesky factorization. We do not differentiate $a_{i j}$ and $A_{i j}$ and always use $A_{i j}$, since i and j imply a unique tile (either $a_{i j}$ or $A_{i j}$ ). In addition, we denote $A_{i j}$ 's submatrix that starts from its local $x$-th row and $y$-th column to its original bottom right

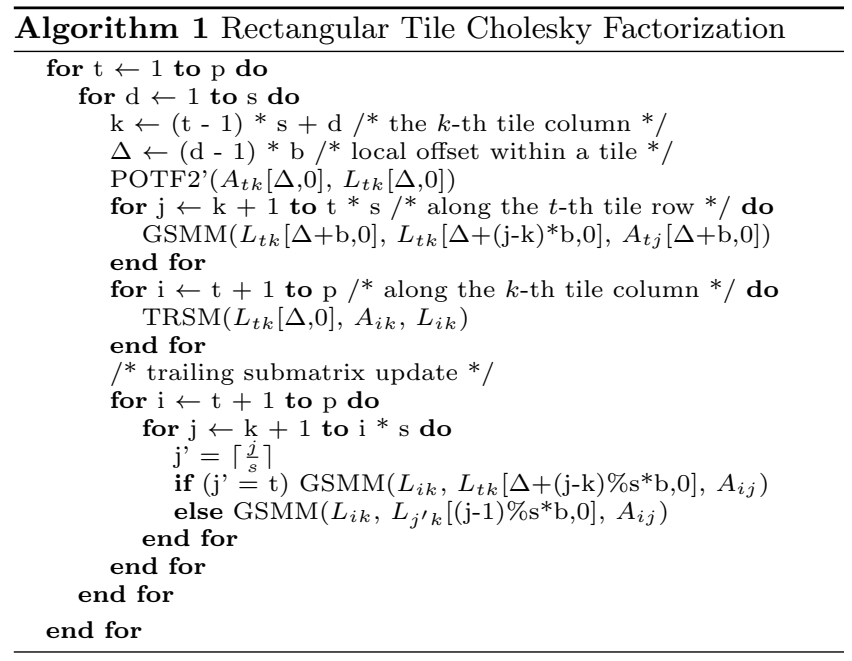

Figure 5: The operations of heterogeneous rectangular tile QR factorization. (a) The matrix A. (b) Compute the QR factorization of $A_{11}$ to get $R_{11}$ and $V_{11}$. (c) Apply $V_{11}$ to update all tiles on the right of $A_{11}$ by calling LARFB. (d) Compute TSQRTs for all tiles below $A_{11}$ to solve $V_{21}$ and $V_{31}$. (e) Apply SSRFBs to update all tiles on $V_{21}$ and $V_{31}$ 's right hand side. (f) After the 1st iteration, we have solved the $R$ factors on the first row with a hight equal to $R_{11}$ 's size. At the 2 nd iteration, we repeat performing (b), (c), (d), (e) on the trailing submatrix that starts from the 2 nd tile column.

corner by $A_{i j}[x, y]$. We denote $A_{i j}[0,0]$ by $A_{i j}$ for short.

Algorithm 1 invokes the same set of kernels as the algorithm for the tile Cholesky factorization 14 except for the kernel POTF2':

- POTF2' $\left(A_{t k}, L_{t k}\right)$ : Given a matrix $A_{t k}$ of size $m \times n$ and $m \geq n$, we let $A_{t k}=\left(\begin{array}{c}A_{t k 1} \\ A_{t k 2}\end{array}\right)$, where $A_{t k 1}$ is of size $n \times n$, and $A_{t k 2}$ is of $(m-n) \times n$. Similarly we let $L_{t k}$ $=\left(\begin{array}{l}L_{t k 1} \\ L_{t k 2}\end{array}\right)$. POTF2' computes $\left(\begin{array}{c}L_{t k 1} \\ L_{t k 2}\end{array}\right)$ by solving $L_{t k 1}=$ $\operatorname{Choleksy}\left(A_{t k 1}\right)$ and $L_{t k 2}=A_{t k 2} L_{t k 1}^{-T}$.

- $\operatorname{TRSM}\left(L_{t k}, A_{i k}, L_{i k}\right)$ computes $L_{i k}=A_{i k} L_{t k}^{-T}$.

- $\operatorname{GSMM}\left(L_{i k}, L_{j k}, A_{i j}\right)$ computes $A_{i j}=A_{i j}-L_{i k} L_{j k}^{T}$.

Figure 4 illustrates the operations of the heterogeneous rectangular tile Cholesky factorization. It shows a matrix of $3 \times 3$ top-level large tiles (i.e., $p=3$ ), each of which is divided into one small and one big rectangular tiles (i.e., $s=2)$. The algorithm goes through $6(=p \cdot s)$ iterations, where the $k$-th iteration solves a submatrix starting from the $k$-th tile column. Since all iterations apply the same operations to different trailing submatrices, we only show the operations of the first iteration.

\subsection{Rectangular Tile QR Factorization}

Algorithm 2 shows the heterogeneous rectangular tile QR factorization. The rectangular tile $\mathrm{QR}$ factorization uses the following set of kernels that are identical to those used in the tile QR factorization 14. For completeness, we present them briefly here:

- $\operatorname{GEQRT}\left(A_{t k}, V_{t k}, R_{t k}, T_{t k}\right)$ computes $\left(V_{t k}, R_{t k}, T_{t k}\right)$ $=\mathrm{QR}\left(A_{t k}\right)$.

- $\operatorname{LARFB}\left(A_{t j}, V_{t k}, T_{t k}, R_{t j}\right)$ computes $R_{t j}$ $=\left(I-V_{t k} T_{t k} V_{t k}^{T}\right) A_{t j}$.

- $\operatorname{TSQRT}\left(R_{t k}, A_{i k}, V_{i k}, T_{i k}\right)$ computes $\left(V_{i k}, T_{i k}, R_{t k}\right)$ $=\mathrm{QR}\left(\begin{array}{l}R_{t k} \\ A_{i k}\end{array}\right)$. 


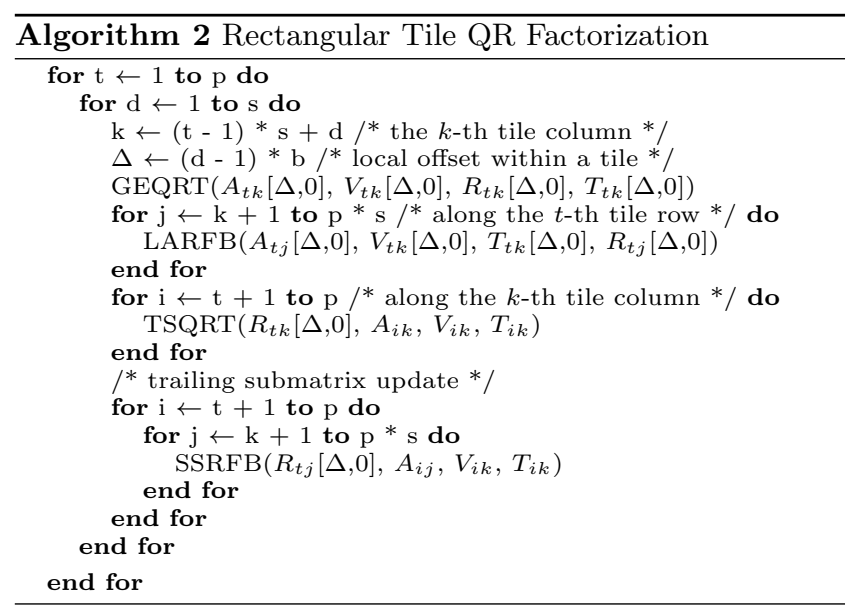
$\begin{aligned} \text { - } & \operatorname{SSRFB}\left(R_{t j}, A_{i j}, V_{i k}, T_{i k}\right) \text { computes }\left(\begin{array}{l}R_{t j} \\ A_{i j}\end{array}\right) \\ = & \left(I-V_{i k} T_{i k} V_{i k}^{T}\right)\left(\begin{array}{c}R_{t j} \\ A_{i j}\end{array}\right) .\end{aligned}$

Figure 5 illustrates the operations of the heterogeneous rectangular tile $\mathrm{QR}$ factorization. It shows a matrix of 3 tile rows and 6 tile columns. The algorithm goes through 6 iterations for the 6 tile columns. Since every iteration performs the same operations on a different trailing submatrix, Fig. 5 only shows the operations of the first iteration.

\subsection{Heterogeneous Block Cyclic Distribution}

We divide a matrix $A$ into $p \times(s \cdot p)$ rectangular tiles using the two-level partitioning method which first partitions $A$ into $p \times p$ large tiles at the top level, then partitions each large tile into $s$ rectangular tiles. Given a multicore and multi-GPU machine, we will distribute $A$ 's tile columns to the host and a number of $P$ GPUs in a 1-D block cyclic way. That is, we statically allocate the $j$-th tile column to $P_{x}$, where $P_{0}$ represents the host system and $P_{x \geq 1}$ represents the $x$-th GPU. We compute $x$ as follows:

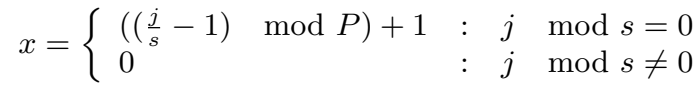

In other words, the columns whose indices are multiples of $s$ are mapped to the $P$ GPUs in a cyclic way and all the other columns go to the single host system.

Figure 6 (a) illustrates a matrix that is divided into rectangular tiles with the two-level partitioning method. Since we always map an entire tile column to either the host or a GPU, the figure omits the boundaries between rows to better illustrate the 1-D method. Figure 6 (b) displays how a matrix with 12 tile columns is allocated to one host and 3 GPUs using the heterogeneous 1-D column block cyclic distribution. The ratio of the sum of $s-1$ rectangular tiles over their remainder controls the workload on the host and on each GPU. Section 4.2 describes a method to determine the ratio for load balancing.

\subsection{Communication Cost}

We consider the heterogeneous system a distributed memory machine such that the host system and the $P$ GPUs represent $P+1$ processes. We also assume the broadcast between processes is implemented by a tree topology in order to make a fair comparison between our algorithms and the ScaLAPACK algorithms 10.

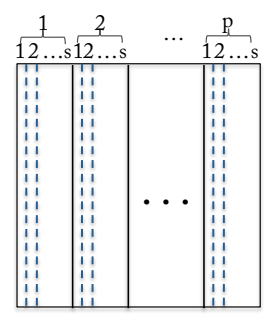

(a)

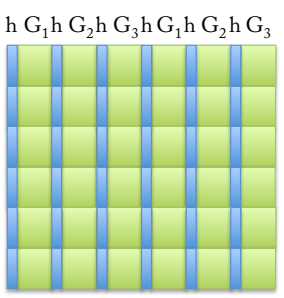

(b)
Figure 6: Heterogeneous 1-D column block cyclic data distribution. (a) The matrix A divided by a two-level partitioning method. $(p, s)$ determines a matrix partition. (b) Allocation of a matrix of $6 \times 12$ rectangular tiles (i.e., $p=6, s=2$ ) to a host and three GPUs: $\mathrm{h}, \mathrm{G}_{1}, \mathrm{G}_{2}$, and $\mathrm{G}_{3}$.

Given a system with one host, $P$ GPUs, and a matrix of size $n \times n$, we partition the matrix into $p \times(p \cdot s)$ rectangular tiles. The small rectangular tile is of size $B \times b$ and $n=$ $p \cdot B$. The number of words communicated by at least one of the processes in the rectangular tile QR (or Cholesky) factorization is bounded by:

$$
\text { Word }=\sum_{k=0}^{p-1}(n-k B) B \log (P+1) \simeq \frac{n^{2}}{2} \log (P)
$$

The communication volume of the rectangular tile algorithm reaches the lower bound of $\Omega\left(\frac{n^{2}}{\sqrt{P}}\right)$ (Theorem 1 to within a factor of $\sqrt{P} \log (P)$. If we use a 2 -D block cyclic distribution instead of the 1-D distribution, we could attain the same communication volume as ScaLAPACK (i.e., $O\left(\frac{n^{2}}{\sqrt{P}} \log P\right)$ 8. 16.). However, it will result in more messages and produce lower performance in practice for the tiled algorithms.

The number of messages sent or received by at least one process in the rectangular QR (or Cholesky) factorization is bounded by:

$$
\text { Message }=\sum_{k=0}^{p-1}(p-k) s \log (P+1) \simeq \frac{p^{2} s}{2} \log (P)
$$

Although the number of messages is larger than that of ScaLAPACK 8, 16 by a factor of $O(p)$, the rectangular tile algorithms have much smaller messages and exhibit a higher degree of parallelism. Note that we want to keep a higher degree of parallelism in order to obtain high performance particularly on many-core systems $3,6,12$.

\section{IMPLEMENTATION}

We have implemented a runtime system to support dataavailability-driven execution where a parent task tries to trigger its children whenever possible. Before the execution starts, we use the heterogeneous 1-D block cyclic method to distribute a matrix across the host and different GPUs statically. Since we have preallocated the $j$-th tile column to the host or a GPU, we require a task modifying the $j$-th tile column be executed by the column's owner (the host or the GPU) to save data movement.

We extend the centralized-version runtime system of our previous work (26] Section 3) to a new one that is suitable for heterogeneous multicore and multi-GPU systems. The 
centralized runtime system works on multicore architectures and has four components (see Fig. 7 as if it had no GPUs):

- Master thread: a single thread that executes a serial program and adds new tasks to the task window.

- Task window: a fixed-size task queue that stores all the generated but unfinished tasks. It is an ordered list that keeps the serial semantic order between tasks.

- Ready task queue: a list of tasks whose inputs are all available. Each node in the list is just a pointer pointing to its corresponding task in the task window.

- Computational threads: every core runs a computational thread. A computational thread picks up a task from the ready task queue whenever it becomes idle. After finishing the task, the thread scans the task window to determine which tasks are the children of the finished task and moves them to the ready task queue.

\subsection{The Extended Runtime System}

Figure 7 shows the architecture of our extended runtime system. Note that the master thread and the task window have not changed.

However, since the host and the GPUs possess disjoint subsets of a matrix, we want to avoid the situation where a task accessing one GPU's data is dispatched to another GPU. Also the task size intended for GPUs is much larger than that intended for CPUs. Therefore, we make the host and each GPU have their own ready task queues. If a ready task modifies a tile that belongs to the host or a GPU, it is sent to the host or the GPU correspondingly.

We have also modified the computational threads. The new runtime system has two types of computational threads: one for CPU cores and the other for GPUs. If a host system has a number of $n$ cores and is connected with $P$ GPUs, the runtime system will launch $P$ computational threads to represent the $P$ GPUs and $(n-P)$ computational threads to represent the remaining CPU cores. Although a GPU computational thread is running on the host, it is able to invoke a GPU kernel automatically as long as the kernel's input is available in the GPU memory.

In the Nvidia CUDA 3.2 programming environment, data movement between different GPUs needs to be relayed by the host. If a host thread is attached to a GPU and allocates a chunk of memory on the GPU, usually only that thread can access the memory. Also there can be only one host thread attached to a GPU at a time. Hence, in our implementation, any data movement to or from a GPU is handled by the GPU's computational thread. With the newly released CUDA 4.0 RC, it is possible to merge several GPU computational threads into one thread.

We create a message box for each GPU computational thread in the host memory. A core computational thread does not have a message box since it cannot access the GPU memory allocated by a GPU computational thread. When moving data either from the host to a GPU or from a GPU to the host, it is the GPU computational thread's responsibility to move the data. We consider three cases to handle the data movement among the host and GPUs:

- Host $\rightarrow$ GPU: after a core computational thread finishes a task, it wants to send the newly modified data

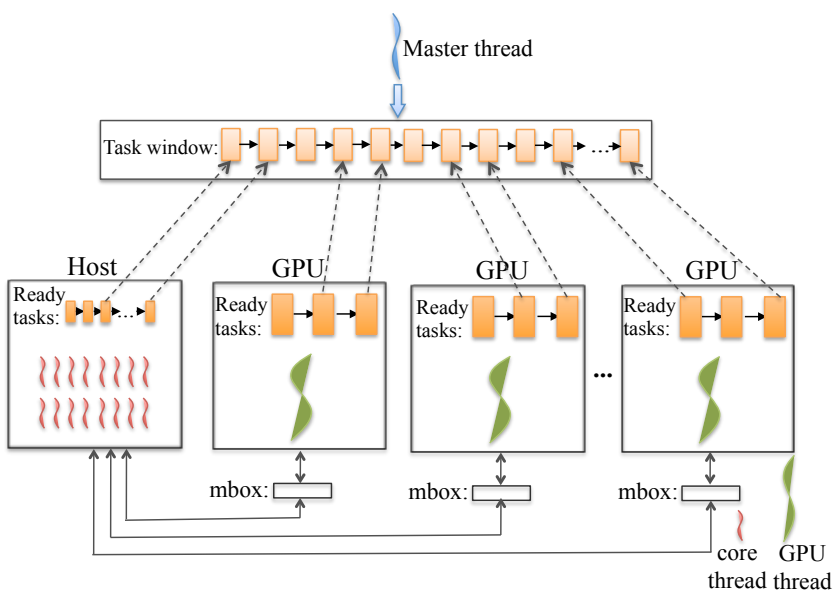

Figure 7: The extended runtime system for heterogeneous multicore and multi-GPU architectures.

to a GPU. The core computational thread adds a message to the GPU's message box telling the GPU to fetch the data.

- GPU $\rightarrow$ Host: after a GPU computational thread finishes a task, the GPU thread adds a message to its own message box and sends the modified data later.

- $\mathrm{GPU}_{s} \rightarrow \mathrm{GPU}_{t}$ : the runtime system generates two messages for this case. First, GPU Gdds a "GPU $_{s} \rightarrow$ Host" message to its own message box with a replay flag to $\mathrm{GPU}_{t}$. When GPU $\mathrm{G}_{s}$ processes the message, it copies the data to the host and then adds a "Host $\rightarrow$ GPU $_{t}$ " message to GPU ${ }_{t}$ 's message box telling GPU to get the data from the host.

Nearly all communications in the Cholesky and QR factorizations are broadcast. During a GPU broadcast, our runtime system copies data only once from the GPU to the host. Then the other GPUs will copy the data from the host to themselves in parallel to minimize communication cost. Note that it is necessary to copy the data to the host for a broadcast operation. A GPU computational thread currently takes charge of both computation and communication and is implemented as follows. With the new CUDA 4.0 RC, it is possible to move process_msg to a dedicated thread to decouple communication from computation.

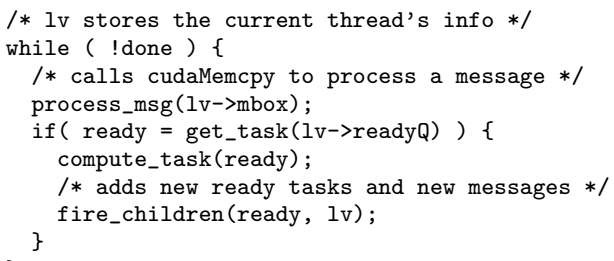

\subsection{Tile Size Tuning}

Load imbalance could happen either between GPUs or between the host and GPUs. We use the 1-D block cyclic distribution method to achieve load balancing between GPUs. Also we adjust the ratio of the CPU tile size to GPU tile size to achieve load balancing between the host and GPUs.

We go through three steps to determine the best tile sizes: 
1. We apply the two-level partitioning method to a matrix and suppose the top-level large tile size $B$ is already given (later we show how to find $B$ ).

2. We use the following formula to estimate the best partition of size $B \times B_{h}$ to be cut off from each top-level tile of size $B \times B$ :

$$
B_{h}=\frac{\text { Perf }_{\text {core }} \cdot \# \text { Cores }}{\text { Perf }_{\text {core }} \cdot \# \text { Cores }+\operatorname{Perf}_{\text {gpu }} \cdot \# G P U s} \cdot B
$$

Perf denotes the maximum performance (in Gflops) of a dominant computational kernel in the algorithm.

3. We start from the estimated size $B_{h}$ and search for an optimal $B_{h}^{*}$ near $B_{h}$. We wrote a script to execute the Cholesky or QR factorization with a random matrix of size $N=c_{0} \cdot B \cdot \# G P U s$. In the implementation, we let $c_{0}=3$ to reduce the searching time. The script adapts the parameter of $B_{h}$ to search for the minimal difference between the host and the GPU computation time. If the host takes more time than a GPU, the script will decrease $B_{h}$ accordingly. This step is inexpensive since the granularity of our fine tuning is 64 for double precision and 96 for single precision due to the significant performance drop when a tile size is not a multiple of 64 or 96 (Fig. 3). In our experiments, it took at most three attempts to find $B_{h}^{*}$.

The top-level tile size $B$ in Step 1 is critical for the GPU performance. To find the best $B$, we search for the minimal matrix size that provides the maximum performance for the dominant GPU kernel (i.e., GEMM for Cholesky and SSRFB for QR). Our search ranges from 128 to 2048 and is performed only once for every new kernel implementation and new GPU architecture. For Nvidia Fermi GPUs, $B$ must be at least 960 for good performance.

Unlike Step 1, Steps 2 and 3 depend on the number of cores and GPUs used in a computation. Note that there are at most (\#Cores - \#GPUs) configurations on a given machine, and not every configuration is useful in practice (e.g., we often use all cores and all GPUs in scientific computing). Later our experimental results show that the auto-tuning method can keep the system load imbalance under $5 \%$ in most cases.

Lemma 1 also justifies our observation that the number of top-level tiles should not affect the load balance between the host and GPUs for the rectangular tile QR factorization.

Lemma 1. Assume a matrix is divided into hybrid rectangular tiles by the two-level partitioning method. Given the performance of the host and GPUs, the partitioning of a large tile into two parts (one for the host and one for GPUs) to keep load balancing, is not related to the number of large tiles for rectangular tile $Q R$ factorizations.

Proof. Suppose there are $P$ GPUs. We let $t_{\text {panel }}^{\text {(host }}$ and $t_{u p}^{\text {(host) }}$ denote the time for the host to compute a panel factorization and a trailing matrix update for a single tile. Similarly, $t_{\text {panel }}^{(g p u)}$ and $t_{u p}^{(g p u)}$ denote the time on a GPU. We assume the kernel computation time does not change much during an execution. So given a matrix partitioned into $p \times p$ large tiles at the top level and assuming $p$ is a multiple of $P$, the execution time of the host is:

$$
T_{\text {host }}=\sum_{i=1}^{p}\left(i \times t_{\text {panel }}^{(\text {host })}+2 i^{2} \times t_{\text {up }}^{(\text {host })}\right) \text {. }
$$

\begin{tabular}{|c|c|c|}
\hline & Host & Attached GPUs \\
\hline Processor type & Intel Xeon X5660 & Nvidia Fermi M2070 \\
\hline Clock rate & $2.8 \mathrm{GHz}$ & $1.15 \mathrm{GHz}$ \\
\hline Processors per node & 2 & 3 \\
\hline Cores per processor & 6 & $14 \mathrm{SMs}$ \\
\hline Memory & 24 GB & $6 \mathrm{~GB}$ per GPU \\
\hline Theo. peak (double) & 11.2 Gflops/core & 515 Gflops/GPU \\
\hline Theo. peak (single) & 22.4 Gflops/core & 1.03 Tflops/GPU \\
\hline Max gemm (double) & 10.7 Gflops/core & 302 Gflops/GPU \\
\hline Max gemm (single) & 21.4 Gflops/core & 635 Gflops/GPU \\
\hline Max ssrfb (double) & 10.0 Gflops/core & 223 Gflops/GPU \\
\hline Max ssrfb (single) & 19.8 Gflops/cores & 466 Gflops/GPU \\
\hline BLAS/LAPACK lib & Intel MKL 10.3 & CUBLAS 3.2, MAGMA \\
\hline Compilers & Intel compilers 11.1 & CUDA toolkit 3.2 \\
\hline OS & CentOS 5.5 & Kernel module 260.19 .14 \\
\hline System interface & - & PCIe x 16 Gen 2 \\
\hline
\end{tabular}

Table 1: Experiment Environment

And the execution time of each GPU is:

$$
T_{\text {gpu }}=\sum_{i=1}^{p}\left(i \times t_{\text {panel }}^{(\text {gpu }}+2 \frac{i^{2}}{P} \times t_{\text {up }}^{(\text {host })}\right) .
$$

It is easy to see that $T_{\text {host }}=T_{\text {gpu }}$ is not related to the number of large tiles $p$. Similarly we can reach the same conclusion for the rectangular tile Cholesky factorization.

\section{PERFORMANCE EVALUATION}

We have implemented the heterogeneous rectangular tile Cholesky and QR factorizations in double precision and single precision. In this section, we present their performance data in weak scalability and strong scalability, respectively. We then measure their load imbalance for three different configurations. We also analyze the efficiency of the runtime system. For every experiment, we have verified that its numerical result is correct.

We conducted experiments on a single node of the heterogeneous Keeneland system at the Oak Ridge National Laboratory. On the system, each node has two Intel Xeon X5660 (Westmere) hexa-core processors and three Nvidia Fermi M2070 GPUs. Table 1 lists the hardware and software resources used in our experiments. The table also lists the maximum performance of gemm and ssrfb used by Cholesky factorization and QR factorization, respectively. The kernel performance serves as an upper bound for the whole program's performance.

\subsection{Weak Scalability}

We use weak scalability to evaluate the capability of a program to solve potentially larger problems when more computing resources are available. In the weak scalability experiment, we increase the input size accordingly when we increase the number of cores and GPUs.

Figure 8 shows the performance of Cholesky and QR factorizations in double precision and single precision, respectively. The $\mathrm{x}$-axis shows the number of cores and GPUs used in the experiment. The y-axis shows Gflops-per-core or Glfops-per-GPU on a logarithmic scale. In each subfigure there are five curves: two "theoretical peak" labels to denote the theoretical peak performance from a single core or from a GPU, one "max GPU-kernel" to denote the maximum GPU kernel performance in the Cholesky or QR factorization which is the upper bound for the whole program, "our perf per core" to denote the performance of our program 


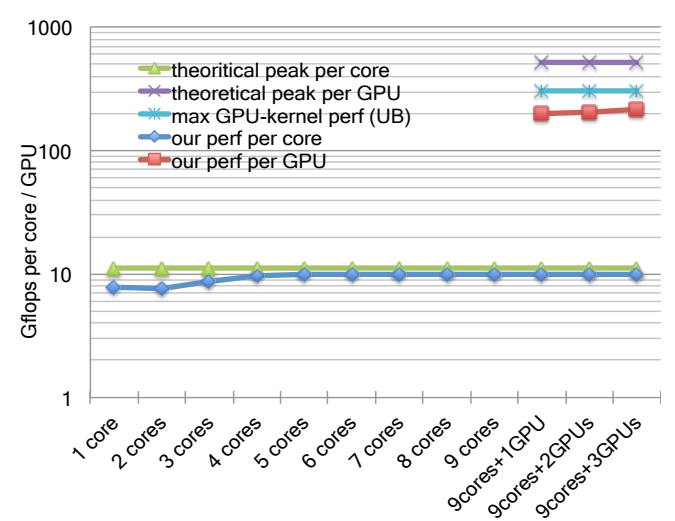

(a) Cholesky in double precision

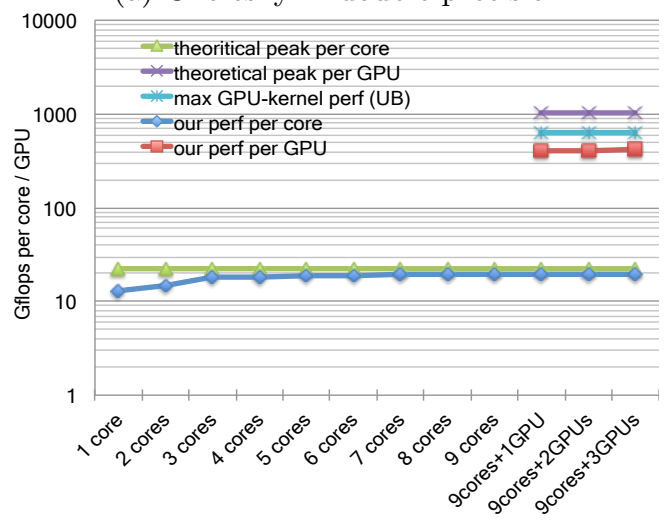

(c) Choleksy in single precision

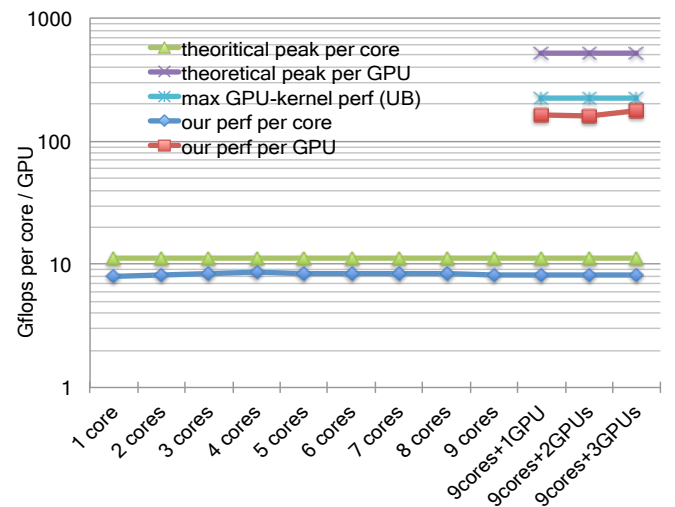

(b) QR in double precision

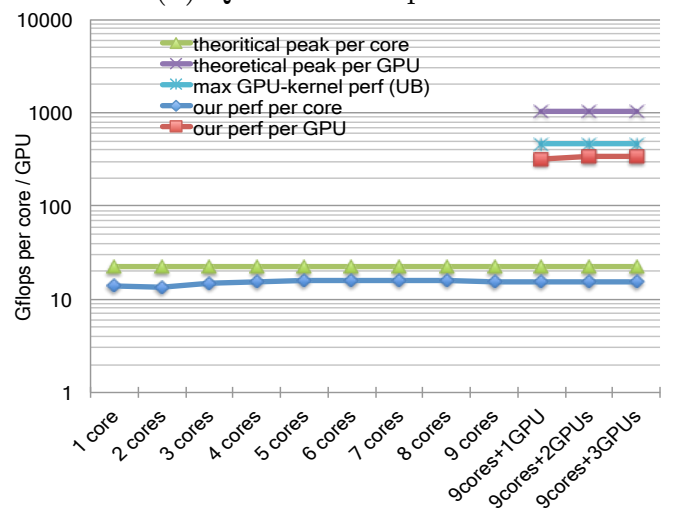

(d) QR in single precision

Figure 8: Weak scalability. The input size increases too while adding more cores and GPUs. The y-axis is presented on a logarithmic scale. OverallPerformance $=\left(\right.$ Perf $_{\text {per_core }} * \#$ cores $)+\left(\right.$ Perf $_{\text {per_gpu }} * \#$ gpus $)$. Note that ideally the performance per core or per GPU should be a flat line.

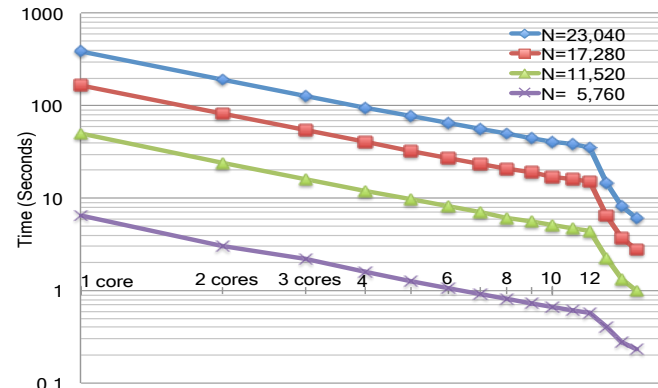

(a) Cholesky in double precision

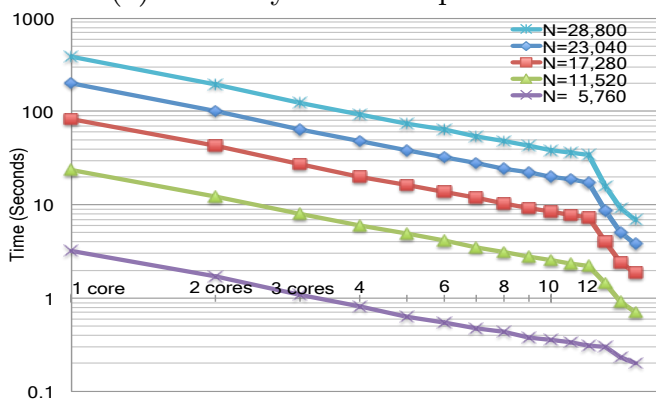

(c) Cholesky in single precision

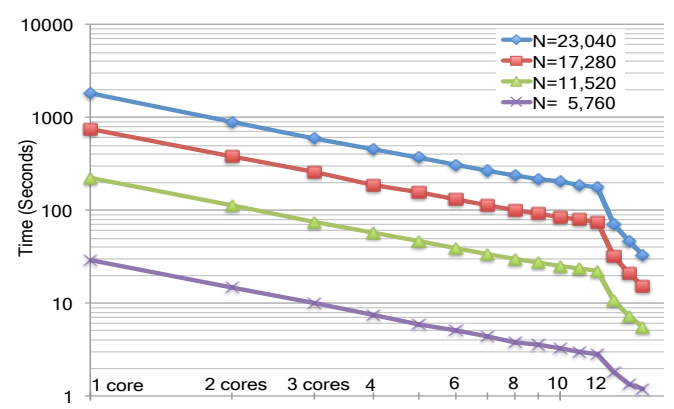

(b) QR in double precision

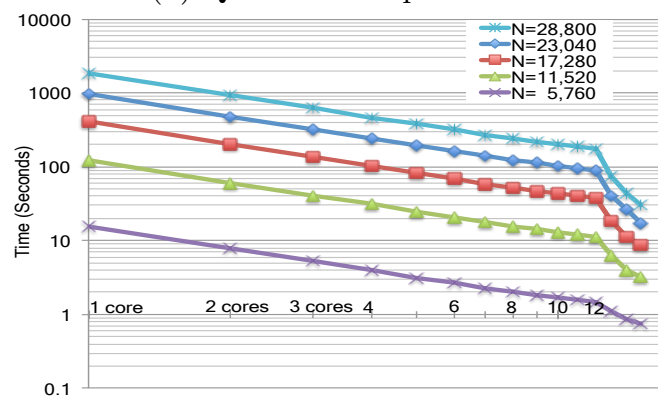

(d) QR in single precision

Figure 9: Strong scalability. The last three ticks on the x-axis (after 12 cores) are: 11 cores +1 GPU, 10 cores +2 GPUs, and 9 cores +3 GPUs. The input size is fixed while adding more cores and GPUs. Both the x-axis and the y-axis are presented on a logarithmic scale. Note that ideally a strong scalability curve should be a straight line in a log-log graph. 
on each CPU core, and "our perf per GPU" to denote our program performance on each GPU.

In the experiments, we first increase the number of cores from 1 to 9 . Then we add 1, 2, and 3 GPUs to the 9 cores. The input sizes for the double precision experiments (i.e., (a), (b)) are: 1000, 2000, . ., 9000, followed by 20000 , 25000 , and 34000 . The input sizes for single precision (i.e., (c), (d)) are the same except for the last three sizes that are 30000,38000 , and 46000. From Fig. 8, we can see that Cholesky and QR factorizations are scalable on both CPU cores and GPUs. Note that ideally the performance per core (or per GPU) is a flat line.

The overall performance of Cholesky factorization or QR factorization can be derived by summing up (perf-per-core $\times$ NumberCores) and (perf-per-gpu $\times$ NumberGPUs). For instance, the double precision Cholesky factorization using 9 cores and 3 GPUs attains an overall performance of 742 Gflops, which is $74 \%$ of the upper bound and $45 \%$ of the theoretical peak. Similarly, the single precision Cholesky factorization has an overall performance of 1.44 Tflops, which is $69 \%$ of the upper bound and $44 \%$ of the theoretical peak. Moreover, the overall performance of $\mathrm{QR}$ factorization is $79 \%$ of the upper bound in double precision, and $73 \%$ of the upper bound in single precision.

\subsection{Strong Scalability}

We use strong scalability to evaluate how much faster a program can solve a specific problem if a user is provided with more computing resources. In the experiment, we measure the execution time to solve a number of matrices each with a different size. Given a fixed-size matrix, we keep adding more computing resources to solve it.

Figure 9 shows the wall clock execution time of Cholesky and QR factorizations in double precision and single precision, respectively. Each graph has several curves, each of which corresponds to a matrix of size $N$. The x-axis shows the number of cores and GPUs on a logarithmic scale. That is, we solve a matrix of size $N$ using $1,2, \ldots, 12$ cores, followed by 11 cores +1 GPU, 10 cores +2 GPUs, and 9 cores +3 GPUs. The y-axis shows execution time in seconds also on a logarithmic scale. Note that an ideal strong scalability curve should be a straight line in a log-log graph.

In Fig. 9 (a), we reduce the execution time of Cholesky factorization in double precision from 393 seconds to 6 seconds for $N=23,040$, and from 6.4 to 0.2 seconds for $N=5,760$. In (b), we reduce the execution time of QR factorization in double precision from 1790 to 33 seconds for $N=23,040$, and from 29 seconds to 1 second for $N=5,760$. Similarly, (c) and (d) display the performance in single precision. In (c), we reduce the execution time of Cholesky factorization from 387 to 7 seconds for $N=28,800$, and from 3.2 to 0.2 seconds for $N=5,760$. In (d), we reduce the execution time of $\mathrm{QR}$ factorization from 1857 to 30 seconds for $N=28,800$, and from 16 to 0.7 seconds for $N=5,760$.

\subsection{Load Balancing}

In this section, we use the metric imbalance_ratio to evaluate the quality of our load balancing, where imbalance_ratio $=\frac{\text { MaxLoad }}{\text { AvgLoad }}$ as proposed in 22. We use computational time to represent the load on a host or GPU. In our implementation, we put timers above and below every computational kernel and sum them up to measure the computational time.
Our experiment uses three different configurations: 3 cores +1 GPU, 6 cores +2 GPUs, and 9 cores +3 GPUs. Given an algorithm (either Cholesky or QR factorization), we first determine the top-level tile size, $B$, for the algorithm; then we determine the partitioning size, $B_{h}^{*}$, for each configuration using the auto-tuning method. We apply the tuned tile sizes to various matrices. For simplicity, we let the matrix size be a multiple of $B$ and suppose the number of tile columns is divisible by the number of GPUs. If the number of tile columns is not divisible by the number of GPUs, we can divide its remainder $(\leq$ NumberGPUs- 1$)$ among all GPUs using a smaller chunk.

Figure 11 shows the imbalance ratio for double and single precision factorizations on three configurations. An imbalance ratio of 1.0 indicates a perfect load balancing. We can see that most of the imbalance ratios are less than 5\%. A few of the first columns have an imbalance ratio of up to $17 \%$. This is because their corresponding matrices have too few top-level tiles. For instance, the first column of the 9Cores+3GPUs configuration has a matrix of three top-level tiles (Fig. 11 (c), (f)). We could increase the number of tiles to alleviate this problem by reducing the top-level tile size.

\subsection{Runtime System Efficiency}

This section investigates whether our runtime system can schedule tasks efficiently and how much further we can improve our program performance. We show the execution time breakdown for the double precision Cholesky and QR factorizations to see where their time goes. The single precision results are the same and not shown here.

Figure 10 shows the execution time breakdown of the Cholesky and QR factorization experiments that use 9 cores and 3 GPUs. The time breakdown data is actually collected from the 9 cores+3GPUs experiment in the weak scalability experiments shown in Fig. 8. The corresponding load imbalance ratio for the two experiments are $3 \%$ and $0.1 \%$ respectively. Note that we show the time breakdown for one of the GPUs because of their balanced load.

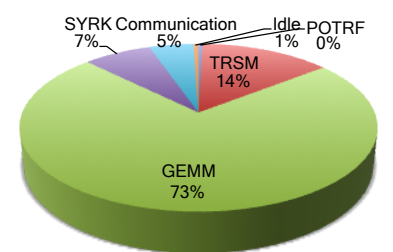

(a) Cholesky factorization

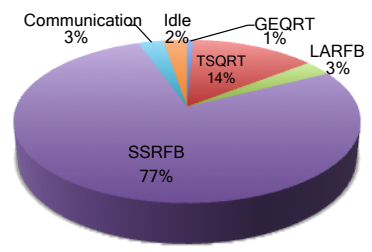

(b) QR factorization
Figure 10: Execution time break down on a GPU for double precision Cholesky and QR factorizations.

As shown in Fig. 10 (a), the double precision Cholesky factorization spends $73 \%$ of its time on kernel GEMM, $14 \%$ on TRSM, $7 \%$ on kernel SYRK, and $5 \%$ on communication. There is only $1 \%$ of idle time. In Fig. 10 (b), all the QR computational kernels take $95 \%$ of the total execution time, the communication takes $3 \%$, and the idle time is $2 \%$. From the analysis, we can see that the runtime system works efficiently and results in little idle time. Also the communication time only occupies a small percentage of time. To further improve the performance, we may need a better implementation of TRSM and TSQRT for GPUs. In addition, the maximum performance of SSRFB for GPUs is only $74 \%$ of the maximum GEMM performance and it needs to be improved too. 


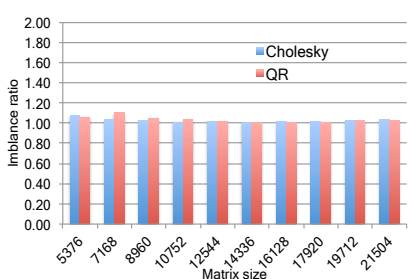

(a) 4 Cores +1 GPU (double)

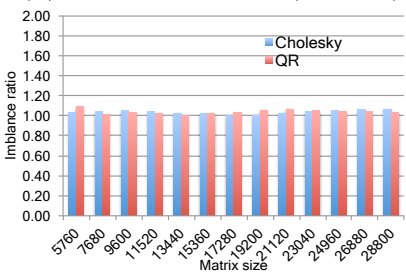

(d) 4 Cores $+1 \mathrm{GPU}$ (single)

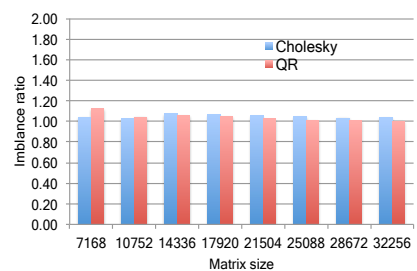

(b) 6 Cores+2GPUs (double)

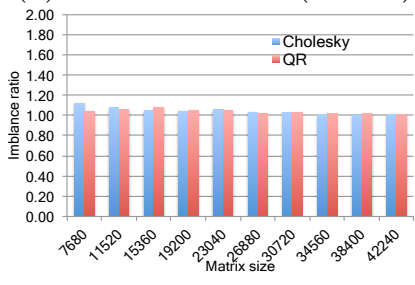

(e) 6Cores+2GPUs (single)

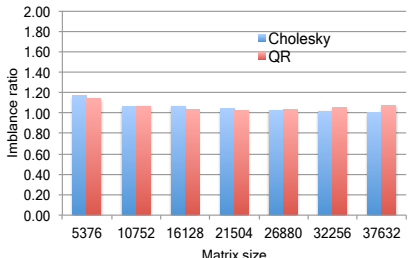

(c) 9 Cores+3GPUs (double)

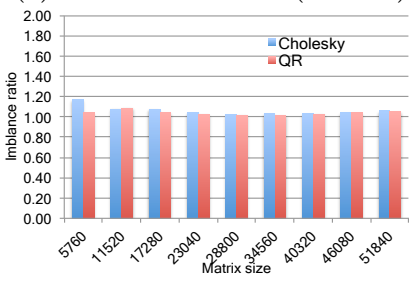

(f) 9Cores+3GPUs (single)

Figure 11: Load imbalance. The metric imbalance_ratio $=\frac{\text { MaxLoad }}{\text { AvgLoad }}$. The closer the ratio is to 1.0 the better.

\section{RELATED WORK}

There are a few linear algebra libraries developed for GPU devices. CUBLAS has implemented the standard BLAS (Basic Linear Algebra Subroutines) library on GPUs 25]. MAGMA and CULA have also implemented a subset of the standard LAPACK library on GPUs 27, 19]. But currently they do not support computations using multiple cores and multiple GPUs.

Demmel et al. have developed the communication-avoiding QR factorization entirely on a single GPU to minimize communication for tall and skinny matrices [5]. By contrast, other GPU implementations (including ours) send panel factorizations back to the host CPUs to compute. Fogue et al. presented a strategy to port the existing PLAPACK library to GPU-accelerated clusters 17. They require that GPUs compute nearly all the compute-intensive work and store all data in GPU memories to reduce communication.

StarSs is a programming model using directives to annotate a sequential source code to execute on various architectures such as SMP, CUDA, and Cell 7]. A programmer is responsible for specifying which piece of code should be executed on a GPU. Then its runtime can execute the annotated code in parallel on the host and GPUs. It uses a software cache mechanism to reduce data transfer cost. Charm ++ is an object-oriented parallel language that uses a dynamic load balancing runtime system to map objects to processors dynamically 21. StarPU also develops a dynamic load balancing framework to execute a sequential code on the host and GPUs in parallel and has been applied to the QR factorization 1. By contrast, we use a simpler static data distribution to minimize communication and to attain high performance simultaneously for matrix computations.

There are many researchers who have studied how to apply static data distribution strategies to heterogeneous distributed memory systems. Dongarra et al. designed an algorithm to map a set of uniform tiles to a 1-D collection of heterogeneous processors 11. Robert et al. proposed a heuristic 2-D block data allocation to extend ScaLAPACK to work on heterogeneous clusters 9]. Lastovetsky et al. developed a static data distribution strategy that takes into account both processor heterogeneity and memory heterogeneity for dense matrix factorizations 23 .

\section{CONCLUSION AND FUTURE WORK}

Developing new parallel software on the heterogeneous multicore and multi-GPU architecture is a challenging task due to a variety of reasons. These reasons include processor heterogeneity, memory heterogeneity, many cores, distributed memory spaces, and an increasing gap between computational performance and communication bandwidth. In order to provide efficient support for matrix computations, we are focused on the objectives of fine-granularity (for a high degree of parallelism), minimized synchronization, minimized communication, and load balancing.

In this paper, we present the heterogeneous algorithms with hybrid tiles to provide high degree of parallelism and cope with processor heterogeneity. We treat the multicore and multi-GPU system as a distributed-memory machine and deploy a heterogeneous block cyclic data distribution to optimize communication. We also introduce an autotuning method to determine the best tile sizes that not only attain high performance, but also achieve load balancing. Furthermore, we have implemented a new runtime system for the heterogeneous multicore and multi-GPU architectures. Although we have applied our approach to the domain of matrix computations, the same methodology and principles can be applied to other scientific applications on multicore and multi-GPU architectures (e.g., heterogeneous algorithms with hybrid tasks, two-level partitioning, a distributed-memory perspective on GPUs, adapting efficient algorithms on clusters, auto-tuning, and so on).

In our approach, the largest matrix size is constrained by the memory capacity of each GPU since we use a static block cyclic distribution method. An approach to solving this issue is to use an "out-of-core" algorithm such as the left looking algorithm, to compute a matrix panel by panel 15. Other future work includes applying the heterogeneous algorithms to clusters with heterogeneous multicore and multiGPU nodes by distributing the top-level tiles to nodes in a 2-D block cyclic way.

\section{Acknowledgment}

We are very grateful to Bonnie Brown and Samuel Crawford for their assistance with this paper. 


\section{REFERENCES}

[1] E. Agullo, C. Augonnet, J. Dongarra, M. Faverge, H. Ltaief, S. Thibault, and S. Tomov. QR factorization on a multicore node enhanced with multiple GPU accelerators. In Proceedings of IEEE International Parallel and Distributed Processing Symposium (IPDPS 2011), Alaska, USA, 2011.

[2] E. Agullo, J. Dongarra, B. Hadri, J. Kurzak, J. Langou, J. Langou, H. Ltaief, P. Luszczek, and A. YarKhan. PLASMA Users' Guide. Technical report, ICL, UTK, 2010.

[3] E. Agullo, B. Hadri, H. Ltaief, and J. Dongarrra. Comparative study of one-sided factorizations with multiple software packages on multi-core hardware. In Proceedings of the Conference on High Performance Computing Networking, Storage and Analysis, SC '09, pages 20:1-20:12, New York, NY, USA, 2009. ACM.

[4] E. Anderson, Z. Bai, C. Bischof, L. Blackford, J. Demmel, J. Dongarra, J. D. Croz, A. Greenbaum, S. Hammarling, A. McKenney, and D. Sorensen. LAPACK Users' Guide. SIAM, 1992.

[5] M. Anderson, G. Ballard, J. Demmel, and K. Keutzer. Communication-avoiding QR decomposition for GPUs. Technical Report UCB/EECS-2010-131, EECS Department, University of California, Berkeley, October 2010.

[6] K. Asanovic, R. Bodik, B. C. Catanzaro, J. J. Gebis, P. Husbands, K. Keutzer, D. A. Patterson, W. L. Plishker, J. Shalf, S. W. Williams, and K. A. Yelick. The landscape of parallel computing research: A view from Berkeley. Technical Report UCB/EECS-2006-183, EECS Department, University of California, Berkeley, December 2006.

[7] E. Ayguadé, R. M. Badia, F. D. Igual, J. Labarta, R. Mayo, and E. S. Quintana-Ortí. An extension of the StarSs programming model for platforms with multiple GPUs. In Proceedings of the 15th International Euro-Par Conference on Parallel Processing, Euro-Par '09, pages 851-862, Berlin, Heidelberg, 2009. Springer-Verlag.

[8] G. Ballard, J. Demmel, O. Holtz, and O. Schwartz. Communication-optimal parallel and sequential Cholesky decomposition. In Proceedings of the twenty-first annual symposium on Parallelism in algorithms and architectures, SPAA '09, pages 245-252, New York, NY, USA, 2009. ACM.

[9] O. Beaumont, V. Boudet, A. Petitet, F. Rastello, and Y. Robert. A proposal for a heterogeneous cluster ScaLAPACK (dense linear solvers). IEEE Transactions on Computers, 50:1052-1070, 2001.

[10] L. S. Blackford, J. Choi, A. Cleary, E. D'Azevedo, J. Demmel, I. Dhillon, J. Dongarra, S. Hammarling, G. Henry, A. Petitet, K. Stanley, D. Walker, and R. Whaley. ScaLAPACK Users' Guide. SIAM, 1997.

[11] P. Boulet, J. Dongarra, Y. Robert, and F. Vivien. Static tiling for heterogeneous computing platforms. Parallel Computing, 25(5):547 - 568, 1999.

[12] A. Buttari, J. Dongarra, J. Kurzak, J. Langou, P. Luszczek, and S. Tomov. The impact of multicore on math software. In Proceedings of the 8th international conference on Applied parallel computing: state of the art in scientific computing,
PARA'06, pages 1-10, Berlin, Heidelberg, 2007. Springer-Verlag.

[13] A. Buttari, J. Langou, J. Kurzak, and J. Dongarra. Parallel tiled QR factorization for multicore architectures. Concurr. Comput. : Pract. Exper., 20(13):1573-1590, 2008.

[14] A. Buttari, J. Langou, J. Kurzak, and J. Dongarra. A class of parallel tiled linear algebra algorithms for multicore architectures. Parallel Comput., 35(1):38-53, 2009.

[15] E. F. D'Azevedo, F. DÕazevedo, and J. J. Dongarra. The design and implementation of the parallel out-of-core ScaLAPACK LU, QR and Cholesky factorization routines, 1997.

[16] J. W. Demmel, L. Grigori, M. F. Hoemmen, and J. Langou. Communication-optimal parallel and sequential QR and LU factorizations. LAPACK Working Note 204, UTK, August 2008.

[17] M. FoguÖ, F. D. Igual, E. S. Quintana-ortŠ, and R. V. D. Geijn. Retargeting PLAPACK to clusters with hardware accelerators. FLAME Working Note 42, 2010.

[18] L. Grigori, J. W. Demmel, and H. Xiang. Communication avoiding Gaussian elimination. In Proceedings of the 2008 ACM/IEEE conference on Supercomputing, SC '08, pages 29:1-29:12, Piscataway, NJ, USA, 2008. IEEE Press.

[19] J. R. Humphrey, D. K. Price, K. E. Spagnoli, A. L. Paolini, and E. J. Kelmelis. CULA: Hybrid GPU accelerated linear algebra routines. In SPIE Defense and Security Symposium (DSS), April 2010.

[20] D. Irony, S. Toledo, and A. Tiskin. Communication lower bounds for distributed-memory matrix multiplication. J. Parallel Distrib. Comput., 64:1017-1026, September 2004.

[21] P. Jetley, L. Wesolowski, F. Gioachin, L. V. Kalé, and T. R. Quinn. Scaling hierarchical N-body simulations on GPU clusters. In Proceedings of the 2010 ACM/IEEE International Conference for High Performance Computing, Networking, Storage and Analysis, SC '10, pages 1-11, Washington, DC, USA, 2010. IEEE Computer Society.

[22] Z. Lan, V. E. Taylor, and G. Bryan. A novel dynamic load balancing scheme for parallel systems. J. Parallel Distrib. Comput., 62:1763-1781, December 2002.

[23] A. Lastovetsky and R. Reddy. Data distribution for dense factorization on computers with memory heterogeneity. Parallel Comput., 33:757-779, December 2007.

[24] J. Nickolls and W. J. Dally. The GPU computing era. IEEE Micro, 30:56-69, March 2010.

[25] NVIDIA. CUDA CUBLAS Library, 2010.

[26] F. Song, A. YarKhan, and J. Dongarra. Dynamic task scheduling for linear algebra algorithms on distributed-memory multicore systems. In SC'09: Proceedings of the Conference on High Performance Computing Networking, Storage and Analysis, pages 1-11, New York, NY, USA, 2009. ACM.

[27] S. Tomov, R. Nath, P. Du, and J. Dongarra. MAGMA Users' Guide. Technical report, ICL, UTK, 2010. 